\title{
A scoping review on the key elements and effects of cardiovascular disease management programmes based on community-based participatory research
}

Bohyun Park ( $\nabla$ bhpark@changwon.ac.kr)

Changwon National University

Juhyeon Yang

Changwon National University

\section{Research Article}

Keywords: Community-Based Participatory Research, Community Participation, Cardiovascular Diseases, Review, Program Development

Posted Date: September 16th, 2021

DOI: https://doi.org/10.21203/rs.3.rs-354020/v1

License: @ (i) This work is licensed under a Creative Commons Attribution 4.0 International License. Read Full License 


\section{Abstract}

Background. This scoping review aims to analyse the literature on community based participatory research (CBPR) -based cardio vascular disease (CVD) management programmes, examining the key elements of their development and implementation and exploring their effectiveness.

Methods. This study is based on the scoping review methodology, consisted of six stages: 1) identifying the research question; 2) identifying relevant studies -search strategy; 3) study selection; 4) charting the data; 5) collating, summarising, and reporting the results; and 6) consultation exercise. The electronic databases used for the literature search were PubMed, Cochrane, and CINAHL. The search period was from 4 March to 3 April 2020 . We selected studies that: 1) were published after 2000;2) targeted community residents over the age of 18; and 3) proposed a CBPR-based CVD management programme, described its development process, or evaluated its effects based on its application. The data were extracted independently by two researchers according to the standardised form.

Results. Among the key elements of such programmes, there were many cases in which community organisations played the leading role in establishing partnerships and cases in which a decision-making committee was formed. Regarding the application of the CBPR principles, community partners participated only in executing the research and not in the process of analyzing and interpreting research results. In addition, In all six RCT studies, the experimental groups showed significantly positive effects compared to the control groups.

Conclusion. It is necessary to develop strategies to improve the criteria based on which CBPR principles cannot be implemented during the development and implementation of a CBPR-based CVD management programme. In addition, more attempts to verify the effectiveness of high-quality research methodology should be made when evaluating the effectiveness of programmes.

\section{Background}

According to the World Health Organization, cardiovascular disease (CVD) is one of the most common causes of death, with 18 million annual deaths globally [1], and poses enormous burden to global health and economy [2]. In many countries, the mortality rate of CVD is higher among people from the lower socioeconomic strata [3-4]. Such differences in the mortality rate of CVD according to income level arise because the factors affecting the occurrence of CVD or resultant mortality differ depending on the income level $[3,5-6]$. Specifically, access to resources promotes healthy behaviour and is regarded as a key social determinant of health [7]. Children from families of low socioeconomic status are more likely to live in areas where such access and opportunities are scarce [8]. In other words, unequal distribution of social determinants of health leads to inequalities in the overall health levels. The need to reduce health inequalities is an indisputable agenda, for which various efforts are being made worldwide. Community-based participatory research (CBPR) is proposed as one such effort.

CBPR developed as an approach that equally engages community members, organisational representatives, and academic researchers in research [9]. The core of CBPR lies in establishing equal cooperative health promotion partnerships among community participants based on equality of participation among the different members of society [10]. As the equality participants independently identify problems and come up with ideas for solutions in the community, it grants the method validity and sustainability, rendering it particularly useful in practice [11]. Such a process can help establish and reinforce the network of related organisations in the local community and improve leadership, which in turn can enhance the autonomous and sustainable capacity for health promotion in the local community [12]. While 'participation' from members of the local community is emphasised during the CBPR process, similar weight is placed on the concept of 'practice'. In other words, CBPR is a concept that integrates both the process of achievement using community capacity and networks as well as the practice-oriented activities [13]. Therefore, CBPR can effectively act as a liaison in the dissemination of academic research results to health project sites.

The community capacity that CBPR aims to foster is regarded as a factor mediating the gap between socioeconomic factors and disparities in health levels, which is among the mechanisms of health inequalities. The CBPR-based health promotion programme is based on these mechanisms of health determinants and aims to reinforce community capacity using mediating variables such as social networks, social support, and social capital, thereby eliminating health inequalities and improving health status. In particular, in order to reduce risk factors for chronic disease such as CVD and eliminate health inequality caused by it, more emphasis is being placed on the importance of the social determinants of health, which is defined as the social, environmental, and related factors in which an individual is born, grows, and resides [14]. The American Heart Association pointed out that 'the most significant opportunities for reducing deaths and disability from CVD in the United States lie with addressing the social determinants of cardiovascular outcomes' [15]. Therefore, the CBPR-based CVD management programme is expected to improve CVD health status compared to existing programmes. However, as community capacity cannot be reinforced in a short span of time, it may take a considerable amount of time to achieve an improved health status. Nevertheless, reinforced community capacity cannot be easily lost and is, therefore, expected to have long-lasting effects, unless the members of the community change.

Reviews of studies implementing CBPR in the field of health care revealed that they targeted specific population groups (Hispanic, African-American, AsianAmerican, immigrants) $[16,17]$ or residents living in specific regions such as Sub-Saharan Africa and islands in the Asia Pacific [18, 19] and focused on the mechanisms of resident participation. Meanwhile, the literature is lacking in reviews that focus on synthesising and presenting the effects of CBPR-based health programmes.

Our scoping review aims to analyse the literature on CBPR-based CVD management programmes, examining the core elements of their development and implementation and exploring their effectiveness.

\section{Methods}


This study is based on the scoping review methodology developed by Arcsey and O'Malley [20] and modified by Levac et al. [21]. It consists of six stages: 1) identifying the research question; 2) identifying relevant studies-search strategy; 3) study selection; 4) charting the data; 5) collating, summarising, and reporting the results; and 6) consultation exercise. In the process of conducting this scoping review and reporting the results, we abided by the PRISMA extension for scoping reviews checklist [22].

\section{Stage 1: Identifying the research questions}

The research questions in this scoping review were formulated and divided into two categories: 1) the 'process' aspect of developing and implementing a CBPR-based CVD management programme, explored through a preliminary survey; and 2) the 'results' aspect of developing and implementing the programme. The research questions are as follows:

1) What are the key elements to be considered in the process of developing and implementing a CBPR-based CVD management programme?

2) How is the CBPR-based CVD management programme structured, and what are the effects?

\section{Stage 2: Identifying relevant studies-the search strategy}

The electronic databases used for the literature search were PubMed, Cochrane, and CINAHL. The search period was from 4 March to 3 April 2020.

The search terms were selected by reviewing the main terms used in the abstract or main text of relevant studies. Based on the characteristics of search field for each electronic database, a specific search strategy was established. The search strategies used across all the electronic databases are as follows: 1) The 'population' search words include 'cardiovascular disease', 'vascul* disease', and 'vascular disease' and were combined using the Boolean operator OR; 2) The 'intervention' search words include 'community-based participatory research', 'participatory action research', 'CBPR', 'PAR', 'participat*', 'community engagement', 'community involvement', 'civic engagement', and 'engagement*' and were combined using the Boolean operator OR; 3 ) The population and intervention search results were combined using the Boolean operator AND; 4) For a comprehensive search, a Medical Subject Headings (MeSH) search was first performed for all search words, which was followed by a search performed in the title/abstract field; 5) The search results were saved after removing duplicate literature using a bibliographic management software (Endnote V.9.3.3.); 6) The search results were shared among the authors.

\section{Stage 3: Study selection}

The relevant studies identified using the search terms proposed in the literature search strategy were reviewed according to the study selection criteria. We selected studies that: 1) were published after 2000;2) targeted community residents over the age of 18; and 3) proposed a CBPR-based CVD management programme, described its development process, or evaluated its effects based on its application. We did not impose any restrictions on the type of research design. Studies other than original research, such as protocols, reviews, and guidelines, studies on inpatients, and studies not written in English were excluded. A study was selected if two researchers reached a consensus after independently screening the study title and abstract. When it was difficult to select a study based solely on the abstract, the full text was reviewed to make a final decision. When the two researchers disagreed, the study selection was made only after sufficient discussion.

A flow chart of the study selection process is shown in Fig. 1. Among the 731 studies identified during the preliminary search of electronic databases, 72 duplicate studies were removed. Upon reviewing the titles and abstracts of the remaining 659 studies, 525 studies were excluded from the analysis because they did not meet the selection criteria. We selected 27 studies after reviewing the full text of 134 studies; the remaining 107 studies did not meet the selection criteria. The following groups of studies were derived from the same research and hence, are considered as one study: 1) Balcazar, Rosenthal et al. [23], Balcazar, de Heer et al. [24], and Balcazar, Wise et al. [25]; 2) Brewer, Balls-Berry et al. [26] and Brewer, Morrison et al. [27]; 3) Brewer, Hayes, Caron et al. [28] and Brewer, Hayes, Jenkins et al. [29]; and 4) Ralston, Lemacks et al. [30], Ralston, Young-Clark et al. [31], and Ralston, Wickrama et al. [32]. Finally, the number of studies included in the review was 21.

Figure 1. The sequential process of identifying the studies included in the analysis

\section{Stage 4: Charting the data}

After the data were extracted independently by two researchers according to the standardised form, the results of the extraction process were compared. A data extraction form was prepared before the start of data extraction and was adjusted as references were compiled on a pilot basis. Through regular research meetings for 2-3 hours twice a week, the results of data extraction were checked, and the degree of agreement was compared.

\section{Stage 5: Collating, summarising, and reporting the results}

For the results regarding Research Question 1-the key factors to be considered in the process of developing and applying a CBPR-based CVD management programme-we summarised common characteristics observed in selected studies, such as partnership establishment (the subject of research initiation and the structure of partnership) and the process of development and application of a CVD management programme (the degree of CBPR principle application, application of planning model and theory, and field activists). We referred to the CBPR quality assessment tool developed by Viswanathan et al. [33] and revised by Chen et al. [34] for the degree of CBPR principle applications, subsequently employing the questions recommended by the latter. The tool modified by Chen et al. [34] consists of two questions as the primary criteria of assessment-'Was the community partner identified?' and 'Was the community partner involved in the planning and/or execution of research?'-and three questions for the secondary criteria-'Was the community partner involved in selection of research topic or development(or review) of the programme?', 'Was the community partner involved in analysis and/or interpretation of research?', and 'Was the community partner involved in dissemination of research results?'. Each question is measured on a three-point scale ( 1 = 'poor'; 2 = 'fair'; and 3 = 'good'). Since all studies reported that there were community partners, the question on whether community partners were identified was removed. Regarding the 
question on whether the community partner was involved in selection of research topic or development (or review) of the programme, we considered participants from the community, who participated in the process of selecting a topic or changing an existing programme to suit their characteristics to be community partners. The results were summarised by evaluating each question, not on the aforementioned three-point scale, but on a simplified two-point scale (yes and no).

For Research Question 2-'How is the CBPR-based CVD management programme structured and what is its effects?'-we summarised the study design, characteristics of participants and intervention programmes, measurement scales, and results. The effects of the programme were listed in the order of study design [single group pre-post, non-randomised controlled trial (nRCT), randomised controlled trial (RCT), and qualitative study] and were described qualitatively.

\section{Stage 6: Consultation exercise}

In order to achieve the purpose of this scoping review, the authors received expert advice on the results derived through the final step of the scoping review, namely reviewing. Three experts with experience in CBPR were asked to review the results. The experts suggested their opinions on whether the situation or experience in CBPR sites was properly reflected in the review result, and the researchers reflected the opinions of the experts during the process of data extraction and summarising of results.

\section{Results}

\section{General characteristics of selected studies}

First, the selected 21 studies were divided into the following main areas of research: purpose, method, country in which the study was conducted, participants, and health problems of participants (Table 1). As for the purpose of study, there were four studies (19.0\%) on the development process of a CBPR-based CVD management programme, three studies (14.3\%) on the development, investigation, and evaluation of a CBPR-based CVD management programme, and 14 studies (66.7\%) that evaluated a CBPR-based CVD management programme. As for the research method, quantitative studies accounted for the largest proportion, with nine studies (42.9\%), followed by six qualitative studies (28.6\%) and six mixed-methods studies (28.6\%). As for the race of the participants in the study, studies on African Americans accounted for the largest proportion (8; 38.1\%), followed by four studies on Hispanics (19.0\%), two studies on Africans (9.5\%), and one study on Asians (4.8\%). In addition, six studies (28.6\%) did not mention the race of participants. There were 15 studies (71.4\%) on adult males and females, one study (4.8\%) on adults and children, and two studies (9.5\%) on only adult females. The remaining three studies (14.3\%) did not mention the age of the participants. In terms of the participant selection criteria, there was one study (4.8\%) on healthy subjects without any underlying disease and eight studies (38.1\%) on subjects with underlying diseases. The underlying disease was further classified into high blood pressure, overweight or obesity, diabetes, and other CVD risk factors.

Table 1

General characteristics of selected study

\begin{tabular}{|c|c|c|}
\hline & & $(\mathrm{N}=21)$ \\
\hline & & $\mathrm{n}(\%)$ \\
\hline \multirow[t]{3}{*}{ Purpose of study } & Developing process of CVD management program & $4(19.0)$ \\
\hline & Development and effectiveness evaluation of CVD management program & $3(14.3)$ \\
\hline & Effectiveness evaluation of CVD management program & 14(66.7) \\
\hline \multirow[t]{3}{*}{ Research methodology } & Quantitative study & $9(42.9)$ \\
\hline & Qualitative study & $6(28.6)$ \\
\hline & Mixed method & $6(28.6)$ \\
\hline \multirow[t]{5}{*}{ Race } & African American (AA) & $8(38.1)$ \\
\hline & Hispanic & $4(19.0)$ \\
\hline & African & $2(9.5)$ \\
\hline & Asian & $1(4.8)$ \\
\hline & Nor reported & $6(28.6)$ \\
\hline \multirow[t]{4}{*}{ Participants } & Adults & 15(71.4) \\
\hline & Adults and children & $1(4.8)$ \\
\hline & Women & $2(9.5)$ \\
\hline & Nor reported & $3(14.3)$ \\
\hline \multirow[t]{3}{*}{ Health problem of participants } & Healthy people & $1(4.8)$ \\
\hline & Having one or more Cardio vascular risk factors & $8(38.1)$ \\
\hline & Nor reported & $12(57.1)$ \\
\hline
\end{tabular}

Page $4 / 18$ 


\section{Key elements of CBPR-based CVD management programme Establishing partnership}

Subject of research initiation

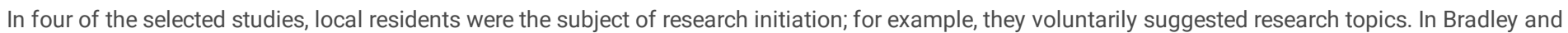

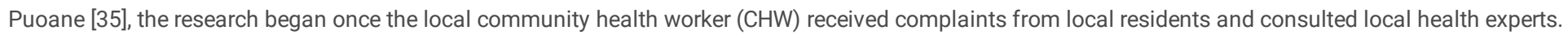

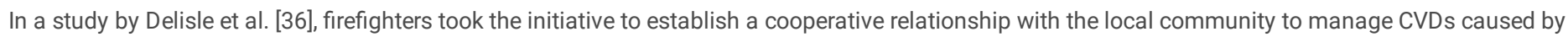

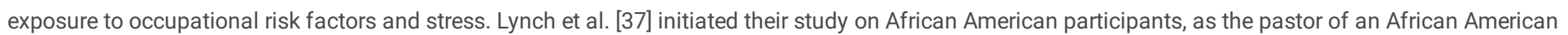

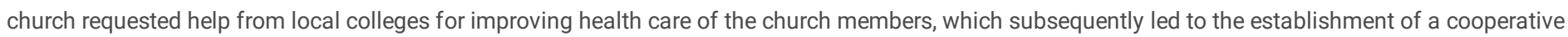

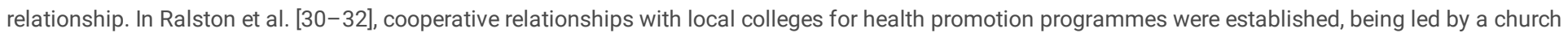
in North Florida.

Partnership establishment status

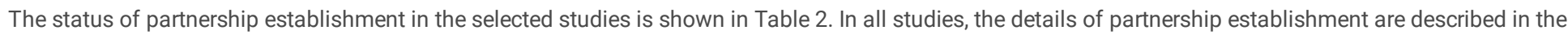

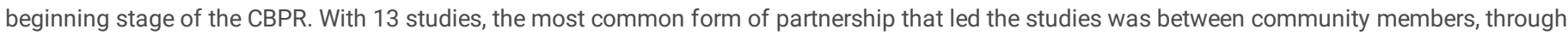

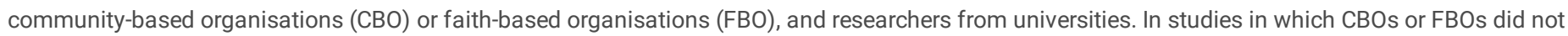

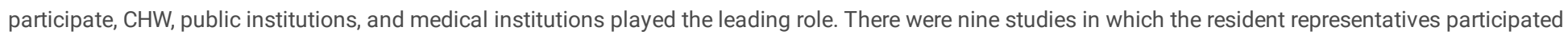

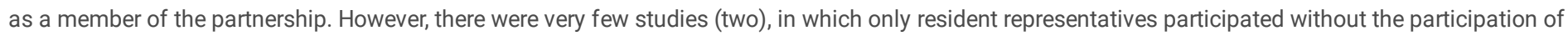

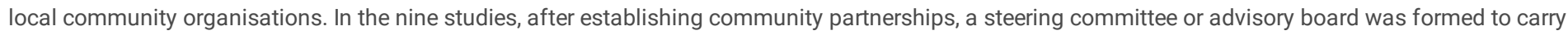

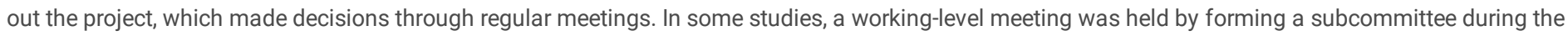
programme development process. 
Table 2

Establishing partnership

\begin{tabular}{|c|c|c|c|c|c|c|c|c|c|}
\hline & & \multicolumn{8}{|c|}{ Community partner } \\
\hline & & University & СBO & FBO & $\begin{array}{l}\text { Public } \\
\text { organisation }\end{array}$ & others & $\begin{array}{l}\text { Community } \\
\text { members }\end{array}$ & $\begin{array}{l}\text { Committee } \\
\text { (or } \\
\text { advisory } \\
\text { board) }\end{array}$ & $\begin{array}{l}\text { Community } \\
\text { activist }\end{array}$ \\
\hline \multirow[t]{19}{*}{ CBO/FBO + University } & $\begin{array}{l}\text { Balcazar, Rosenthal et al. } \\
2009 \text { [23] }\end{array}$ & 0 & 0 & & & hospital & & 0 & promotores \\
\hline & $\begin{array}{l}\text { Balcazar, de Heer et al. } \\
2010 \text { [24] }\end{array}$ & & & & & & & & \\
\hline & $\begin{array}{l}\text { Balcazar, Wise et al. } 2012 \\
\text { [25] }\end{array}$ & & & & & & & & \\
\hline & $\begin{array}{l}\text { Brewer, Hayes, Caron et } \\
\text { al. } 2019 \text { [28] }\end{array}$ & 0 & & 0 & & & & & $\begin{array}{l}\text { FAITH } \\
\text { partner }\end{array}$ \\
\hline & $\begin{array}{l}\text { Brewer, Hayes, Jenkins et } \\
\text { al. } 2019 \text { [29] }\end{array}$ & & & & & & & & \\
\hline & $\begin{array}{l}\text { Ralston, Lemacks et al. } \\
2014 \text { [30] }\end{array}$ & 0 & & 0 & & & 0 & & \\
\hline & $\begin{array}{l}\text { Ralston, Young-Clark et al. } \\
2017 \text { [31] }\end{array}$ & & & & & & & & \\
\hline & $\begin{array}{l}\text { Ralston, Wickrama et al. } \\
2020 \text { [32] }\end{array}$ & & & & & & & & \\
\hline & Bess et al. 2019 [59] & 0 & 0 & 0 & & & 0 & 0 & \\
\hline & $\begin{array}{l}\text { Brewer, Balls-Berry et al. } \\
2017 \text { [26] }\end{array}$ & 0 & & 0 & & & & & $\begin{array}{l}\text { FAITH } \\
\text { partner }\end{array}$ \\
\hline & $\begin{array}{l}\text { Brewer, Morrison et al. } \\
2019 \text { [27] }\end{array}$ & & & & & & & & \\
\hline & $\begin{array}{l}\text { Jayaprakash et al. } 2016 \\
\text { [46] }\end{array}$ & 0 & 0 & & & & & 0 & \\
\hline & Kim et al. (2004) [39] & 0 & 0 & & & & & 0 & $\begin{array}{l}\text { lay health } \\
\text { advisors }\end{array}$ \\
\hline & Lynch et al. (2019) [37] & 0 & & 0 & & & 0 & 0 & \\
\hline & $\begin{array}{l}\text { Mudd-Martin et al. (2013) } \\
\text { [38] }\end{array}$ & 0 & 0 & & & & & & promotores \\
\hline & Pazoki et al. (2007) [41] & 0 & 0 & & & & & 0 & $\begin{array}{l}\text { local health } \\
\text { volunteers }\end{array}$ \\
\hline & Pinsker et al. (2017) [60] & 0 & & 0 & & & 0 & & \\
\hline & Schulz et al. (2015) [42] & 0 & 0 & 0 & & & 0 & & $\begin{array}{l}\text { community } \\
\text { health } \\
\text { promotors }\end{array}$ \\
\hline & Skolarus et al. (2018) [45] & 0 & & 0 & & & & & \\
\hline \multirow[t]{6}{*}{ Others + University } & $\begin{array}{l}\text { Bradley \& Puoane (2007) } \\
\text { [35] }\end{array}$ & 0 & & & & $\mathrm{CHW}$ & & & \\
\hline & $\begin{array}{l}\text { Chimberengwa \& Naidoo } \\
\text { (2019) [44] }\end{array}$ & & & & 0 & nurse & 0 & 0 & $\begin{array}{l}\text { Village } \\
\text { health } \\
\text { workers }\end{array}$ \\
\hline & Delisle et al. (2013) [36] & 0 & & & 0 & & 0 & & $\begin{array}{l}\text { peer fitness } \\
\text { trainer }\end{array}$ \\
\hline & Rorie et al. (2011) [40] & 0 & & & 0 & & & & $\begin{array}{l}\text { resident } \\
\text { health } \\
\text { advocates }\end{array}$ \\
\hline & $\begin{array}{l}\text { Schroeder et al. (2017) } \\
\text { [61] }\end{array}$ & 0 & & & & & 0 & & \\
\hline & Folta et al. (2019) [62] & 0 & & & & HE & & & local leader \\
\hline \multirow{2}{*}{$\begin{array}{l}\text { Community members } \\
\text { + University }\end{array}$} & Perry et al. (2017) [63] & 0 & & & & & 0 & 0 & \\
\hline & Zoellner et al. (2014) [43] & 0 & & & & & 0 & 0 & $\begin{array}{l}\text { walking } \\
\text { coach }\end{array}$ \\
\hline
\end{tabular}


CBO: Community-based organization

FBO: Faith-based organization

CHW: Community health worker

HE: Health educator

\section{Development and implementation of intervention programmes}

Application of CBPR principles

CBPR quality evaluation criteria (whether community partners participated in the process of planning and conducting research, selection and modification of research topics, and analysis/interpretation and dissemination of research results) were used to analyse how consistently the CBPR principles were applied during the process of development and implementation of intervention programmes (Table 3 ). While it has been observed that community partners participated in the process of conducting research in all studies, they participated in the process of research planning only in 16 studies. Among 16 studies in which community partners participated in the research topic selection or modification process, community partners and researchers participated in the research topic selection process in 11 studies; in the remaining five studies, community partners participated in modifying the previously developed programme to suit the needs of local residents. There was no study in which community partners participated in the analysis and interpretation of the research results. In 10 studies, community partners participated in the process of disseminating the research results. Among them, community partners participated as co-authors of research papers in eight studies, in addition to disseminating research results to local residents through flyers or newspapers in the only three studies. 
Table 3

Application of CBPR principle

\begin{tabular}{|c|c|c|c|c|c|c|c|}
\hline \multirow[t]{2}{*}{ Purpose of study } & \multirow[t]{2}{*}{ Author(Year) } & \multicolumn{2}{|c|}{ Planning/Execution } & \multicolumn{2}{|c|}{$\begin{array}{l}\text { Topic } \\
\text { selection/Adaptation }\end{array}$} & \multirow[t]{2}{*}{ Analysis/Implementation } & \multirow[t]{2}{*}{ Dissemination } \\
\hline & & Planning & Execution & $\begin{array}{l}\text { Topic } \\
\text { selection }\end{array}$ & Adaptation & & \\
\hline \multirow[t]{4}{*}{ Development process } & $\begin{array}{l}\text { Bess et al. } \\
2019 \text { [59] }\end{array}$ & 0 & 0 & & 0 & NA & NA \\
\hline & $\begin{array}{l}\text { Bradley \& } \\
\text { Puoane (2007) } \\
\text { [35] }\end{array}$ & 0 & 0 & 0 & & NA & NA \\
\hline & $\begin{array}{l}\text { Anthony et al. } \\
\text { (2013) [36] }\end{array}$ & 0 & 0 & 0 & & NA & NA \\
\hline & $\begin{array}{l}\text { Perry et al. } \\
\text { (2017) [63] }\end{array}$ & 0 & 0 & & 0 & NA & NA \\
\hline \multirow[t]{15}{*}{ Application/ Evaluation } & $\begin{array}{l}\text { Brewer, Balls- } \\
\text { Berry et al. } \\
2017 \text { [26] }\end{array}$ & 0 & 0 & & 0 & & \\
\hline & $\begin{array}{l}\text { Brewer, } \\
\text { Morrison et al. } \\
2019 \text { [27] }\end{array}$ & & & & & & \\
\hline & $\begin{array}{l}\text { Chimberengwa } \\
\text { \& Naidoo } \\
\text { (2019) [44] }\end{array}$ & & 0 & & & & \\
\hline & $\begin{array}{l}\text { Folta et al. } \\
\text { (2019) [62] }\end{array}$ & & 0 & & & & \\
\hline & $\begin{array}{l}\text { Jayaprakash } \\
\text { et al. (2016) } \\
\text { [46] }\end{array}$ & 0 & 0 & & & & 0 \\
\hline & $\begin{array}{l}\text { Kim et al. } \\
(2004) \text { [39] }\end{array}$ & & 0 & & & & \\
\hline & $\begin{array}{l}\text { Lynch et al. } \\
(2019) \text { [37] }\end{array}$ & 0 & 0 & 0 & & & 0 \\
\hline & $\begin{array}{l}\text { Mudd-Martin } \\
\text { et al. } 2013 \text { [38] }\end{array}$ & 0 & 0 & 0 & & & \\
\hline & $\begin{array}{l}\text { Pazoki et al. } \\
\text { (2007) [41] }\end{array}$ & & 0 & 0 & & & $0 *$ \\
\hline & $\begin{array}{l}\text { Pinsker et al. } \\
\text { (2017) [60] }\end{array}$ & 0 & 0 & 0 & & & 0 \\
\hline & $\begin{array}{l}\text { Rorie et al. } \\
(2011) \text { [40] }\end{array}$ & & 0 & & & & \\
\hline & $\begin{array}{l}\text { Schroeder et } \\
\text { al. (2017) [61] }\end{array}$ & 0 & 0 & 0 & & & 0 \\
\hline & $\begin{array}{l}\text { Schulz et al. } \\
\text { (2015) [42] }\end{array}$ & 0 & 0 & 0 & & & 0 \\
\hline & $\begin{array}{l}\text { Skolarus et al. } \\
\text { (2018) [45] }\end{array}$ & 0 & 0 & 0 & & & $0,0 *$ \\
\hline & $\begin{array}{l}\text { Zoellner et al. } \\
\text { (2014) [43] }\end{array}$ & 0 & 0 & 0 & & & 0 \\
\hline \multirow[t]{3}{*}{ Development/Application/Evaluation } & $\begin{array}{l}\text { Balcazar, } \\
\text { Rosenthal et } \\
\text { al. } 2009 \text { [23] }\end{array}$ & 0 & 0 & & 0 & & $0 *$ \\
\hline & $\begin{array}{l}\text { Balcazar, de } \\
\text { Heer et al. } \\
2010 \text { [24] }\end{array}$ & & & & & & \\
\hline & $\begin{array}{l}\text { Balcazar, Wise } \\
\text { et al. } 2012 \text { [25] }\end{array}$ & & & & & & \\
\hline
\end{tabular}




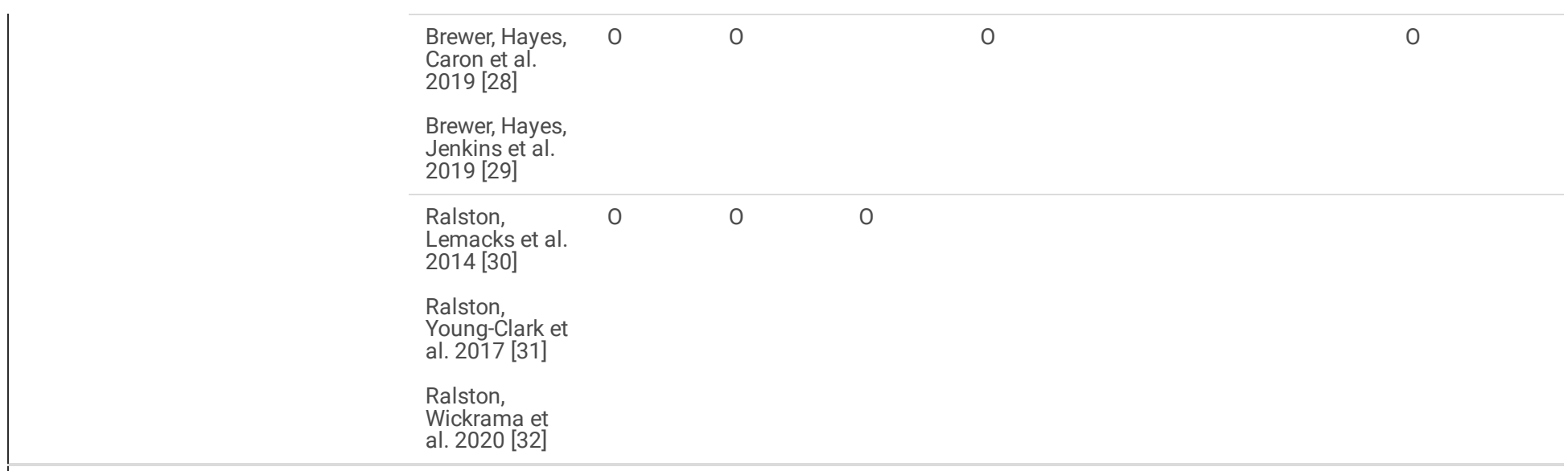

* Community partners disseminate program results to local residents through flyers or news

Application of a planning model or health-related theory to the program development

Twelve of the selected studies applied a health planning model or health-related theory during the development process of a CVD management programme. Six studies applied planning models: three studies with intervention mapping, one with the precede-proceed model, one with the CATCH-PATH model, and one with the chronic care model.

Seven studies applied a health-related theory, two of which applied two or more theories in combination: four studies applied self-determination theory; three studies, socio-ecological theory; and two studies, transtheoretical models. The theory of planned behaviour, social cognitive theory, health belief model, and theories on self-efficacy, social support, motivation, and social norms were applied in the studies.

Use of field activists

In 12 of the selected studies, community activists were recruited to run the programmes. Among them, five studies presented the details of activist education programmes. In a programme for Hispanics, activists (called 'promotores') were mainly recruited [23-25, 38]. Promotores were selected from among those with participation history in community projects and were asked to obtain health-related qualifications within 30 days of employment. They were trained for a total of 45 hours over two weeks on CVD risk factor reduction methods, capacity reinforcing strategies, community resource identification, advocacy, and data collection methods. Community partners participated in the development of education programmes for promotores. In a study by Kim et al. [39], a Lay Health Advisor (LHA) was recruited to provide a total of 13 education sessions, which took place twice a week (3 hours per session), on physical activity, maintenance of smoking cessation environment, and healthy eating habits. During the last two education sessions, LHAs were given an opportunity to demonstrate methods of practice and personal training. Since LHAs lacked experience in conducting research projects, they were trained in advance on obtaining consent from participants and on collecting data. Delisle et al. [36] selected eight peer fitness trainers and gave them 40 hours of physical activity training (developed by the researchers' team), conducted motivational interviews for behavioural changes, and provided additional training on behavioural change modelling, goal setting, and techniques to enhance capacities.

However, the remaining seven studies only described the role of activists without the details of activist education programmes. Activists were recruited from among residents who spoke the same language as the residents. Their main roles were participant recruitment [26, 31, 40], resident education [40], operational assistance for programmes [26, 31, 41, 42], and a point of contact between participants and researchers during the programme [43]. Some studies that described how they utilised activists, reported paying salaries to some or all activists so that they can fulfil their roles $[24,25,38,40]$.

\section{The effects of CBPR-based CVD management programmes}

Of the 21 selected studies, 17 studies evaluated the effectiveness of the CBPR-based CVD management programme after implementation (Table 4). 
Table 4

The effectiveness of CVD management program based on CBPR

\begin{tabular}{|c|c|c|c|c|c|c|}
\hline \multirow{3}{*}{$\begin{array}{l}\text { Authors } \\
\text { (year) }\end{array}$} & \multirow{3}{*}{$\begin{array}{l}\text { Study } \\
\text { design }\end{array}$} & \multirow{3}{*}{$\begin{array}{l}\text { Study } \\
\text { participants }\end{array}$} & \multicolumn{4}{|l|}{ Intervention } \\
\hline & & & \multirow{2}{*}{$\begin{array}{l}\text { Session } \\
\text { /Duration }\end{array}$} & \multirow{2}{*}{$\begin{array}{l}\text { Name of } \\
\text { intervention } \\
\text { [detailed contents[ }\end{array}$} & \multirow[t]{2}{*}{$\begin{array}{l}\text { Method and } \\
\text { media }\end{array}$} & \multirow[t]{2}{*}{$\begin{array}{l}\text { Effect (statistical } \\
\text { significance) }\end{array}$} \\
\hline & & & & & & \\
\hline \multirow{3}{*}{$\begin{array}{l}\text { Lynch et al. } \\
2019 \text { [37] }\end{array}$} & \multirow[t]{3}{*}{ pre-post } & age $\geq 18$ & \multirow[t]{3}{*}{2 hours $\star 24$ times $/ 9$ months } & \multirow{2}{*}{$\begin{array}{l}\text { Abundant Living in } \\
\text { Vibrant Energy } \\
\text { (ALIVE) intervention }\end{array}$} & \multirow{3}{*}{$\begin{array}{l}\text { Bible study, small } \\
\text { group sessions, } \\
\text { church-wide } \\
\text { activities, videos, } \\
\text { hand outs, self- } \\
\text { monitor vegetable } \\
\text { consumption, } \\
\text { bulletin }\end{array}$} & \multirow{3}{*}{$\begin{array}{l}\text { Vegetable servings } \\
\text { consumed/day, total diet } \\
\text { quality: (+) } \\
\text { Weight, Blood pressure: } \\
(+)\end{array}$} \\
\hline & & $N=206$ & & & & \\
\hline & & $\begin{array}{l}\text { (mean } \\
\text { age:57.5) }\end{array}$ & & $\begin{array}{l}\text { [nutrition, physical } \\
\text { activity } \square\end{array}$ & & \\
\hline \multirow[t]{3}{*}{$\begin{array}{l}\text { Pinsker et al. } \\
2017 \text { [60] }\end{array}$} & \multirow[t]{3}{*}{ pre-post } & $\begin{array}{l}\text { Participants: } \\
\mathrm{N}=310\end{array}$ & \multirow[t]{3}{*}{12 weeks } & \multirow{3}{*}{$\begin{array}{l}\text { Body and Soul } \\
\text { program } \\
\text { [nutrition }\end{array}$} & \multirow{3}{*}{$\begin{array}{l}\text { BP check, cooking } \\
\text { demonstrations, } \\
\text { peer counseling, } \\
\text { video, handbook, } \\
\text { posters }\end{array}$} & \multirow{3}{*}{$\begin{array}{l}\text { Eating habits(weekly } \\
\text { servings of fruit and } \\
\text { vegetables): (+) } \\
\text { Physical activity: (+) } \\
\text { Peer counseling: }(+)\end{array}$} \\
\hline & & \multirow{2}{*}{$\begin{array}{l}\text { Church } \\
\text { coordinators: } \\
\mathrm{N}=11\end{array}$} & & & & \\
\hline & & & & & & \\
\hline \multirow[t]{3}{*}{$\begin{array}{l}\text { Schroeder et } \\
\text { al. } 2017 \text { [61] }\end{array}$} & \multirow[t]{3}{*}{ pre-post } & $\begin{array}{l}\text { Adult: } N= \\
372\end{array}$ & \multirow{3}{*}{$\begin{array}{l}2 \\
\text { hours/week*2months(spring, } \\
\text { fall)(5 years) }\end{array}$} & \multirow{3}{*}{$\begin{array}{l}\text { Dance for Health } \\
\text { [physical activity }\end{array}$} & \multirow[t]{3}{*}{ line dance } & $\begin{array}{l}\text { Achieved target heart } \\
\text { rate: }(+)\end{array}$ \\
\hline & & $\begin{array}{l}\text { (mean } \\
\text { age:52.4) }\end{array}$ & & & & PACES Scales: (+) \\
\hline & & $\begin{array}{l}\text { Children: } \mathrm{N}= \\
149 \text { (mean } \\
\text { age:12.2) }\end{array}$ & & & & \\
\hline \multirow{3}{*}{$\begin{array}{l}\text { Zoellner et al. } \\
2014 \text { [43] }\end{array}$} & \multirow[t]{3}{*}{ pre-post } & age $\geq 40$ & \multirow[t]{3}{*}{90 minutes $/$ month $* 6$ months } & \multirow{2}{*}{$\begin{array}{l}\text { HUB City } \\
\text { Steps(lifestyle } \\
\text { intervention) }\end{array}$} & \multirow{3}{*}{$\begin{array}{l}\text { peer support, } \\
\text { pedometer, group } \\
\text { physical activity }\end{array}$} & $\mathrm{BP}:(+)$ \\
\hline & & $N=269$ & & & & Sugar intake: (+) \\
\hline & & $\begin{array}{l}\text { (mean } \\
\text { age:44.0) }\end{array}$ & & $\begin{array}{l}\text { [nutrition, physical } \\
\text { activity } \square\end{array}$ & & \\
\hline Balcazar et al. & RCT & age:30 75 & 2 hours/week ${ }^{\star} 2$ months + & Exp: Su Corazón, Su & Exp: telephone & Diastolic BP: (+) \\
\hline & & Exp.: $\mathrm{N}=192$ & & 然 & session & Diet behaviors(lower salt \\
\hline & & $\begin{array}{l}\text { (mean } \\
\text { age:53.5) }\end{array}$ & & Con.: Basic & Con.: material & total cholesterol) : EG (+) \\
\hline & & $\begin{array}{l}\text { Con.: } \mathrm{N}=136 \\
\text { (mean } \\
\text { age:54.0) }\end{array}$ & & $\begin{array}{l}\text { educational } \\
\text { materials }\end{array}$ & & $\begin{array}{l}\text { Health beliefs } \\
\text { (benefits/susceptibility): } \\
\text { EG }(+)\end{array}$ \\
\hline $\begin{array}{l}\text { Folta et al. } \\
2019 \text { [62] }\end{array}$ & RCT & only female & Exp.: 2 times/week*24weeks & $\begin{array}{l}\text { Exp.: Strong Hearts, } \\
\text { Healthy }\end{array}$ & $\begin{array}{l}\text { Exp.: aerobic } \\
\text { exercise, }\end{array}$ & $\begin{array}{l}\text { Diet behaviors(intake of } \\
\text { fruit and vegetables): EG }\end{array}$ \\
\hline & & age $\geq 40$ & Con.: 1 time/month $\star 24$ weeks & $\begin{array}{l}\text { Communities(SHHC) } \\
\text { curriculum }\end{array}$ & $\begin{array}{l}\text { progressive } \\
\text { strength training, }\end{array}$ & $(+)$ \\
\hline & & $\begin{array}{l}\text { Exp.: } \mathrm{N}=101 \\
\text { (mean } \\
\text { age:58.9) }\end{array}$ & & $\begin{array}{l}\text { ¿diet, nutrition, } \\
\text { physical activity }\end{array}$ & $\begin{array}{l}\text { healthy } \\
\text { eating practices, } \\
\text { discussion + }\end{array}$ & $\begin{array}{l}\text { Physical activity(Walking } \\
\text { MET-min/week): } \mathrm{EG}(+)\end{array}$ \\
\hline & & Con.: $\mathrm{N}=93$ & & $\begin{array}{l}\text { Con.: Didactic } \\
\text { healthy lifestyle }\end{array}$ & $\begin{array}{l}\text { engagement) } \\
\text { engaric club (CIVIC }\end{array}$ & \\
\hline & & $\begin{array}{l}\text { (mean } \\
\text { age:59.0) }\end{array}$ & & & $\begin{array}{l}\text { Con.: didactic } \\
\text { approach }\end{array}$ & \\
\hline Pazoki et al. & RCT & only female & 1.5hours/week*8weeks & Exp.: Lifestyle & Exp.: audio-taped, & Systolic BP: EG (+) \\
\hline & & age:25 64 & & program & visits, educational & Physical activity: EG (+) \\
\hline & & $\begin{array}{l}\text { Exp.: } \mathrm{N}=179 \\
\text { (mean } \\
\text { age:39.4) }\end{array}$ & & $\begin{array}{l}\square C V \text { diseases, } \\
\text { smoking, nutrition, } \\
\text { physical activity } \square\end{array}$ & Con.: NR & $\begin{array}{l}\text { Healthy heart knowledge: } \\
\text { EG }(+)\end{array}$ \\
\hline & & Con.: N = 179 & & Con.: NR & & \\
\hline & & $\begin{array}{l}\text { (mean } \\
\text { age:NR) }\end{array}$ & & & & \\
\hline
\end{tabular}




\begin{tabular}{|c|c|c|c|c|c|c|}
\hline \multirow{2}{*}{$\begin{array}{l}\text { Authors } \\
\text { (year) }\end{array}$} & \multirow{2}{*}{$\begin{array}{l}\text { Study } \\
\text { design }\end{array}$} & \multirow{2}{*}{$\begin{array}{l}\text { Study } \\
\text { participants }\end{array}$} & \multicolumn{4}{|l|}{ Intervention } \\
\hline & & & $\begin{array}{l}\text { Session } \\
\text { /Duration }\end{array}$ & $\begin{array}{l}\text { Name of } \\
\text { intervention } \\
\text { [detailed contents[ }\end{array}$ & $\begin{array}{l}\text { Method and } \\
\text { media }\end{array}$ & $\begin{array}{l}\text { Effect (statistical } \\
\text { significance) }\end{array}$ \\
\hline \multirow[t]{4}{*}{$\begin{array}{l}\text { Schulz et al. } \\
2015 \text { [42] }\end{array}$} & \multirow[t]{4}{*}{ RCT } & & \multirow[t]{4}{*}{$\begin{array}{l}3 \text { times }(45 \sim 90 \\
\text { minutes)/week*32weeks }\end{array}$} & \multirow{2}{*}{$\begin{array}{l}\text { Exp.: Walk Your } \\
\text { Heart to } \\
\text { Health(WYHH) } \\
\text { intervention }\end{array}$} & \multirow{4}{*}{$\begin{array}{l}\text { Exp.: contacts, } \\
\text { walking } \\
\text { Con.: lagged } \\
\text { intervention }\end{array}$} & \multirow{4}{*}{$\begin{array}{l}\text { Physical activity(steps, } \\
\text { adherence): (+) } \\
\text { Indicators of } \\
\text { cardiovascular risk: }(+)\end{array}$} \\
\hline & & $\begin{array}{l}\mathrm{N}=695 \\
\text { (mean } \\
\text { age:46.6) }\end{array}$ & & & & \\
\hline & & Exp.: NR & & \multirow{2}{*}{$\begin{array}{l}\text { Con.: lagged } \\
\text { intervention }\end{array}$} & & \\
\hline & & Con.: NR & & & & \\
\hline \multirow{3}{*}{$\begin{array}{l}\text { Rorie et al. } \\
2010 \text { [40] }\end{array}$} & \multirow[t]{3}{*}{ non RCT } & age $\geq 18$ & Exp.: summer(2007, 2008) & $\begin{array}{l}\text { Exp.: Resident health } \\
\text { advocate(RHA) } \\
\text { intervention }\end{array}$ & $\begin{array}{l}\text { Exp.: personal } \\
\text { contacts, flyers }\end{array}$ & \multirow{2}{*}{$\begin{array}{l}\text { Mobile health screening } \\
\text { rates: EG }(+) \\
\text { Follow-up appointment: } \\
(+)\end{array}$} \\
\hline & & $\begin{array}{l}\text { Exp.: } \mathrm{N}=100 \\
\text { (mean } \\
\text { age:45.1) }\end{array}$ & \multirow[t]{2}{*}{ Con.: summer(2007, 2008) } & $\begin{array}{l}\text { intervention } \\
\text { \mobile health } \\
\text { screening } \square\end{array}$ & Con.: flyers & \\
\hline & & $\begin{array}{l}\text { Con.: } \mathrm{N}=47 \\
\text { (mean } \\
\text { age:42.8) }\end{array}$ & & $\begin{array}{l}\text { Con.: standard } \\
\text { recruitment }\end{array}$ & & \\
\hline \multirow{4}{*}{$\begin{array}{l}\text { Brewer, Hayes, } \\
\text { Jenkins et al. } \\
2019 \text { [29] }\end{array}$} & \multirow[t]{4}{*}{ pre-post } & age $\geq 18$ & \multirow[t]{4}{*}{ 10weeks + 28weeks(f/u) } & \multirow{3}{*}{$\begin{array}{l}\text { Fostering African } \\
\text { American } \\
\text { Improvement in } \\
\text { Total Health } \\
\text { (FAITH!) App }\end{array}$} & \multirow{4}{*}{$\begin{array}{l}\text { App-multimedia } \\
\text { education } \\
\text { modules(videos), } \\
\text { self-quizzes, } \\
\text { diet/PA self- } \\
\text { monitoring, social } \\
\text { networking }\end{array}$} & BP: (+) \\
\hline & & $N=50$ & & & & Behaviors(diet, \\
\hline & & $\begin{array}{l}\text { (mean } \\
\text { age:49.6) }\end{array}$ & & & & $\begin{array}{l}\text { Servings/day, Physical } \\
\text { activity): (+) }\end{array}$ \\
\hline & & & & $\begin{array}{l}\text { [nutrition, physical } \\
\text { activity] }\end{array}$ & & LS7 score: $(+)$ \\
\hline \multirow{4}{*}{$\begin{array}{l}\text { Brewer, Balls- } \\
\text { Berry et al. } \\
2017 \text { [26] } \\
\\
\text { Brewer, } \\
\text { Morrison et al. } \\
2019 \text { [27] }\end{array}$} & \multirow{4}{*}{$\begin{array}{l}\text { Qualitative } \\
\text { pre-post }\end{array}$} & age:30 75 & \multirow[t]{4}{*}{$\begin{array}{l}90 \text { minutes } / 2 \text { weeks } * 16 \text { weeks } \\
+3 \text { months }(f / u)\end{array}$} & \multirow{4}{*}{$\begin{array}{l}\text { Fostering African } \\
\text { American } \\
\text { Improvement in } \\
\text { Total Health } \\
\text { (FAITH!) }\end{array}$} & \multirow{4}{*}{$\begin{array}{l}\text { Material, } \\
\text { interactive } \\
\text { lectures, videos, } \\
\text { cooking } \\
\text { demonstrations, } \\
\text { exercise classes }\end{array}$} & $\begin{array}{l}\text { Qn-CV health knowledge: } \\
(+)\end{array}$ \\
\hline & & $N=37$ & & & & LS7 score: (+) \\
\hline & & $\begin{array}{l}\text { (mean } \\
\text { age:51.7) }\end{array}$ & & & & QI-FAITH! \\
\hline & & & & & & $\begin{array}{l}\text { program(research } \\
\text { perception, benefits, } \\
\text { satisfaction): }(+)\end{array}$ \\
\hline \multirow{3}{*}{$\begin{array}{l}\text { Kim et al. } 2004 \\
\text { [39] }\end{array}$} & \multirow{3}{*}{$\begin{array}{l}\text { Qualitative } \\
\text { pre-post }\end{array}$} & age $\geq 18$ & \multirow[t]{3}{*}{2 hours/week*1 month } & \multirow{3}{*}{$\begin{array}{l}\text { Health education } \\
\text { classes } \\
\text { [nutrition, physical } \\
\text { activity, smoke-free[ }\end{array}$} & $\begin{array}{l}\text { Video, audio-tape, } \\
\text { picture cards, }\end{array}$ & $\begin{array}{l}\text { Qn-lifestyle } \\
\text { behaviors(physical }\end{array}$ \\
\hline & & $N=272$ & & & $\begin{array}{l}\text { plastic food } \\
\text { models, poster, } \\
\text { measuring spoons }\end{array}$ & $\begin{array}{l}\text { activity, nutrition } \\
\text { behavior, smoke-free } \\
\text { behavior): }(+)\end{array}$ \\
\hline & & & & & & $\begin{array}{l}\text { Ql-feasibility and } \\
\text { effectiveness of LHAs: } \\
(+)\end{array}$ \\
\hline $\begin{array}{l}\text { Mudd-Martin } \\
\text { et al. } 2013 \text { [38] }\end{array}$ & Qualitative & age $\geq 18$ & 2hours/week*8weeks & $\begin{array}{l}\text { Su Corazon, Su Vida } \\
\text { (Your Heart, Your }\end{array}$ & $\begin{array}{l}\text { Educational } \\
\text { models, deep }\end{array}$ & $\begin{array}{l}\text { Qn-knowledge(CVD and } \\
\text { DM): }(+)\end{array}$ \\
\hline & pre-post & & & & $\begin{array}{l}\text { breathing, imagery } \\
\text { exercises, }\end{array}$ & HPLP II scale: (+) \\
\hline & & $\begin{array}{l}\text { (mean } \\
\text { age:44.5) }\end{array}$ & & $\begin{array}{l}\text { ]cardiovascular } \\
\text { diseases, nutrition, } \\
\text { stress management, } \\
\text { physical activity, } \\
\text { smoke-free }]\end{array}$ & discussions & $\begin{array}{l}\text { QI-program(acceptability, } \\
\text { responsiveness): (+) }\end{array}$ \\
\hline Ralston, & Qualitative & age $\geq 45$ & 6 months +12 months $(f / u)+$ & Exp.: Health for & Exp.: materials, & Qn-diet behaviors(daily \\
\hline $2014[30]$ & $\mathrm{RCT}$ & Exp.: $\mathrm{N}=143$ & & intervention & $\begin{array}{l}\text { promotion } \\
\text { activities }\end{array}$ & fat intake, Fat Screener): \\
\hline $\begin{array}{l}\text { Ralston, } \\
\text { Young-Ćlark et } \\
\text { al. } 2017 \text { [31] }\end{array}$ & & Con.: $\mathrm{N}=157$ & & $\begin{array}{l}\text { पCVD knowledge, } \\
\text { diet, stress } \\
\text { management, } \\
\text { physical activity }]\end{array}$ & Con.: materials & $\begin{array}{l}\text { QI-challenges: providing } \\
\text { consistency in } \\
\text { programming even during }\end{array}$ \\
\hline $\begin{array}{l}\text { Ralston, } \\
\text { Wickrama et } \\
\text { al. } 2020 \text { [32] }\end{array}$ & & & & $\begin{array}{l}\text { Con.: Health ministry } \\
\text { development } \\
\text { activities }\end{array}$ & & $\begin{array}{l}\text { participant absences; } \\
\text { providing structured } \\
\text { activities to assist health }\end{array}$ \\
\hline & & & & $\begin{array}{l}\text { पCVD knowledge, } \\
\text { nutrition, physical } \\
\text { activity, stress }\end{array}$ & & $\begin{array}{l}\text { sustainability; and } \\
\text { addressing changes at } \\
\text { the church level }\end{array}$ \\
\hline & & & & reduction! & & \\
\hline
\end{tabular}




\begin{tabular}{|c|c|c|c|c|c|c|}
\hline \multirow{2}{*}{$\begin{array}{l}\text { Authors } \\
\text { (year) }\end{array}$} & \multirow{2}{*}{$\begin{array}{l}\text { Study } \\
\text { design }\end{array}$} & \multirow{2}{*}{$\begin{array}{l}\text { Study } \\
\text { participants }\end{array}$} & \multicolumn{4}{|l|}{ Intervention } \\
\hline & & & $\begin{array}{l}\text { Session } \\
\text { /Duration }\end{array}$ & $\begin{array}{l}\text { Name of } \\
\text { intervention } \\
\text { [detailed contents }\end{array}$ & $\begin{array}{l}\text { Method and } \\
\text { media }\end{array}$ & $\begin{array}{l}\text { Effect (statistical } \\
\text { significance) }\end{array}$ \\
\hline $\begin{array}{l}\text { Skolarus et al. } \\
2018 \text { [45] }\end{array}$ & $\begin{array}{l}\text { Qualitative } \\
\text { RCT }\end{array}$ & $\begin{array}{l}\text { age } \geq 18 \\
\text { Exp.: } \mathrm{N}=48 \\
\text { (mean } \\
\text { age:57.8) } \\
\text { Con.: } \mathrm{N}=46 \\
\text { (mean } \\
\text { age:58.7) }\end{array}$ & 6 months & $\begin{array}{l}\text { Exp.: Reach } \\
\text { Out(Mobile Health } \\
\text { Technology } \\
\text { Intervention) } \\
\text { Con.: usual care } \\
\text { पhypertension, diet, } \\
\text { physical activity }\end{array}$ & $\begin{array}{l}\text { Exp.: material, BP } \\
\text { self-monitoring, } \\
\text { text messages } \\
\text { Con.: material }\end{array}$ & $\begin{array}{l}\text { Qn-program(Feasibility, } \\
\text { satisfaction): (+) } \\
\\
\text { Ql-Tailored text messages } \\
\text { received unanimous } \\
\text { positive responses. }\end{array}$ \\
\hline $\begin{array}{l}\text { Chimberengwa } \\
\& \text { Naidoo } 2019 \\
{[44]}\end{array}$ & Qualitative & $N=22$ & 1 time/month $* 6$ months & $\begin{array}{l}\text { chronic care } \\
\text { management (CCM) } \\
\text { Thealth education }\end{array}$ & $\begin{array}{l}\text { BP check, peer } \\
\text { support, } \\
\text { counselling, group } \\
\text { discussions }\end{array}$ & $\begin{array}{l}\text { Qn- } \\
\text { knowledge(hypertension): } \\
(+) \\
\text { enroll rate of } \\
\text { hypertension patient: (+), } \\
\text { Pill pickup rate, treatment } \\
\text { compliance: }(+)\end{array}$ \\
\hline $\begin{array}{l}\text { Jayaprakash } \\
\text { et al. } 2016 \text { [46] }\end{array}$ & Qualitative & $\begin{array}{l}\text { age:30 } 60 \\
\mathrm{~N}=31\end{array}$ & $\begin{array}{l}\text { Exp.::60 } 90 \text { minutes/week*6 } \\
\text { weeks }\end{array}$ & $\begin{array}{l}\text { South Asian Heart } \\
\text { Lifestyle Intervention } \\
\text { (SAHELI) } \\
\text { पHeart Disease, } \\
\text { physical activity, } \\
\text { diet, weight, stress } \\
\text { management }\end{array}$ & $\begin{array}{l}\text { Exp.: group } \\
\text { classes, activities, } \\
\text { counseling, } \\
\text { telephone support, } \\
\text { pedometers }\end{array}$ & $\begin{array}{l}\text { Qn-Quality of Life: (+), } \\
\text { behavior change: }(+), \\
\text { health knowledge: }(+), \\
\text { physical activity: }(+) \\
\text { Qn-trusted CBO setting, } \\
\text { culturally concordant } \\
\text { study staff activities } \\
\text { self-monitoring with } \\
\text { pedometers helped. } \\
\text { family involvement and } \\
\text { women-only classes were } \\
\text { beneficial. } \\
\text { reduce participant } \\
\text { burden, needed greater } \\
\text { financial resources. }\end{array}$ \\
\hline \multicolumn{7}{|c|}{$(+)$ : positive effect } \\
\hline \multicolumn{7}{|c|}{ NR: not reported } \\
\hline \multicolumn{7}{|c|}{ QI: results of Qualitative research } \\
\hline $\begin{array}{l}\text { LS7: Life's Simp } \\
\text { pressure (BP), tc } \\
\text { HPLP II scale(he } \\
\text { PACES = Physic }\end{array}$ & $\begin{array}{l}\text { 7, } 4 \text { modifia } \\
\text { al cholesterol } \\
\text { Ith responsib } \\
\text { Activity Enjo }\end{array}$ & $\begin{array}{l}\text { le health beha } \\
\text { and fasting gl } \\
\text { ity, physical a } \\
\text { ment Scale. }\end{array}$ & $\begin{array}{l}\text { rs (physical activity, diet, smoki } \\
\text { ose) } \\
\text { vity, interpersonal relations, stre }\end{array}$ & $\begin{array}{l}\text { g, and body mass index } \\
\text { management) }\end{array}$ & (BMI)) and 3 modifia & le biological factors (blood \\
\hline
\end{tabular}

The studies were categorised by the study design as follows: 5 studies with one group pre-/post test design, 4 RCTs, and 1 non-randomised control group design among quantitative studies, 5 mixed-methods studies, and 2 qualitative studies. As for the age of participants, 10 studies were on adults over the age of 18 years, and 5 studies were on middle-aged people over the age of 30 to 40 years. The duration of the intervention varied from 4 weeks to 36 weeks, with 24 weeks ( 6 months) being the most common ( 5 studies), followed by 8 weeks ( 3 studies). Regarding programme details, 10 studies explored two or more topics: 15 programmes were on nutrition (or diet) and physical activity, 7 on knowledge related to CVD, 3 on smoking-free habits, 3 on stress management, 2 on weight loss, and 1 on mobile health screening. As for how the programme was run, 9 studies were conducted with counselling (personal contacts, home visits, and calls), 3 studies with multimedia-based education (social networking and text messages), 7 studies with lectures (video and audio tape), 3 studies with cooking demonstrations (models), 8 physical activity programmes (walking, dance, exercise classes, treadmill, and pedometer), 6 small group discussions and seminars, 3 posters, and 1 civic engagement (HEART club).

To measure the effectiveness of each CBPR-based CVD management programme Clinical, behavioural, cognitive, and psychological variables were used; 7 studies measured clinical characteristics (BP, total cholesterol, HDL-cholesterol, LDL-cholesterol, weight, and heart rate); 9 studies measured behavioural characteristics (diet, physical activity, smoke-free, pill pickup rate, treatment compliance, screening rates, follow-up appointments, and participation rates); 5 studies measured changes in CVD-related knowledge as cognitive characteristics; and 1 study measured the quality of life based on psychological characteristics. In addition, 4 studies evaluated research perceptions, benefits, satisfaction, acceptability, and feasibility. In 6 of the selected studies with a randomised controlled group, the experimental group showed significantly positive effects compared to the control group.

Page 12/18 
Qualitative studies cited statements from interviews with participants in the CBPR-based CVD management programme to report their positive evaluation of the programmes [44, 45], improved awareness of CVD, and positive changes in health behaviour $[38,39,46]$. Some studies have shown that field activists have the capacity to play an intermediary role between residents and health care professionals [44] and play a key role in ensuring that CBOs recruit and retain participants. In addition, the importance of fostering trust and rapport between programme facilitators and participants as well as the need for family participation were suggested [38,46]. On the other hand, the lack of sustainability of participation was suggested as a limitation [31].

\section{Discussion}

We reviewed the studies published over the last 10 years on CBPR-based CVD management programmes for adults and aimed to examine the key factors and effects of such programmes and provide fundamental evidence for seeking effective interventions in the future.

In the process of designing a study to develop a CVD management program based on CBPR, we judged that it was necessary to grasp how and how much CVD management programs based on CBPR were developed and applied in the community. In order to find answers to these research questions, a scope review was more suitable than a systematic literature review. A scoping review differs from other types of literature review in the sense that it provides information on practice, policy making, and research by mapping the literature on a specific subject or research area to identify the types of evidence, data sources, key concepts, and gaps in the studies [47], and provide maps or snapshots of existing literature without quantitative synthesis or quality assessment [48]. We conducted the scoping review according to the procedure proposed by Arksey and O'Malley [20].

In the process of applying CBPR, the following results were derived. First, CBPR partnership is initiated by local residents, local health experts, or researchers. In the studies selected for this review, there were only four studies in which local residents voluntarily proposed research topics. The fact that community partners have become the subject of research initiation means that they are aware of community health problems and are active in the process of finding alternative solutions. Whether local residents are the subject of a CBPR initiation is considered an important factor, because there is a higher possibility that it can be implemented as an actual health promotion project [13]. Therefore, in-depth exploration of the context of the community and previous experiences is required for the region where the community partner has been the subject of research initiation.

In community-based health research, it is recommended or required to establish and operate a community advisory board or steering committee to reflect and communicate the opinions of community members throughout the research process [49]. In previous studies, the role of the participant organisation was limited to a consultation role in which it relayed the opinions and responses of the community. On the other hand, in CBPRs, these act as a representative organisation that provides key information such as the characteristics, strengths, weaknesses, and needs of the local community and play the role of an equal partner in practice during the decision-making process on a problem at hand [50]. For community participation to be considered as community capacity, a global and solid foundation for participation must be formed. It should depart from the typical participation that merely fills up the positions of participants as a formality and instead, be practical such that it involves cooperative discussions on planning and assessment of community health promotion projects through active participation with a sense of ownership [51]. However, while most of the selected studies have described the details of partnership establishment, there were only a few studies in which community representatives played a leading role. Therefore, it is necessary to provide opportunities for them to participate in research to exert influence on the community.

The key of CBPR is that members of the local community continuously and actively participate as equal partners with health experts and researchers during the entire process, from research planning to evaluation and reflection of results. Such a process enhances autonomous and sustainable community capacity for health promotion [12]. In this study, for the quality assessment of CBPRs in the selected studies, we partially modified the tool, which was originally developed by Viswanathan et al. [33] and modified by Chen et al. [34], and lays out the core principles of community participation research. In all the studies selected in this study, community partners partially participated during the process of conducting research. However, there were relatively few studies in which they participated in the process of planning and selecting or modifying the research topic. In the case of studies in which they did not participate during the process of planning, but during conducting, the quality of CBPR can be evaluated as low, as community partners mostly participated passively by recruiting participants or assisting in the programme. In particular, none of the studies involved community partners in the analysis or interpretation of the research results. It is necessary to develop strategies, including the development of evaluation tools, to allow community partners to be effectively involved in the process of evaluating research results. A total of 10 studies were conducted in which community partners participated in the dissemination of research results. In three of them, community partners participated in disseminating the research results to local residents through leaflets or newspapers. In the existing conventional health care research system, the low rate of practical implementation has been criticised [52]. However, since CBPR places an emphasis on 'practice' as its core element and has an advantage of offering a possibility of participatory linkages that facilitates dissemination of academic research results to the field, the Prevention Research Center (PRC) of Centers for Disease Control and Prevention (CDC) requires its programme to perform more than one CBPR per core research area $[53,54]$. Nevertheless, according to the results of our study, there were very few studies in which community residents participated in the wide dissemination of research results to the community, which is considered one of the merits of CBPR. While an ideal study would satisfy all five criteria of CBPR quality evaluation, none of the selected studies did so. Since inputs from community residents on health projects can make an important contribution to the health promotion and welfare improvement of the local community and act as an important factor in disease prevention [55], it is necessary to understand the practical reasons why it is difficult for community members to participate in the entire research process from an equal position and apply the true CBPR principles.

Cook [13] proposed to use two complementary methods, a qualitative research method using participatory observation and a quantitative research method using data analysis for effective CBPR research. Among selected studies, quantitative studies were the most at 10 . Next, there were six mixed method studies, and five qualitative studies. CBPR is a process-centred research in which qualitative research methods have been proven to be particularly useful, as it emphasises the process of conversation and discussion between community members, researchers, and representatives of public-private organisations, in order to share an understanding of the significance and background of current health problems in the community [12]. In addition, despite having the same research topic, its need and significance can be perceived differently depending on the context and characteristics of each community; a combination of

Page $13 / 18$ 
quantitative and qualitative research methods should be used to address this issues. Therefore, the use of mixed-methods research approaches can well reflect the characteristics of CBPR that exhibit a cyclical structure in the process of identifying and solving various problems in communities [13].

An RCT is the gold standard in terms of generating scientific evidence in clinical research; it is conducted with the most rigorous and thorough research methods to explain the relationship between the intervention and an outcome and can provide the highest level of evidence [56]. However, most of the studies selected in our review were nRCTs or pre-post studies; only six studies were RCTs. In the future, to clarify the effectiveness of interventions on CBPR-based CVD management programmes and apply the best evidence to practice, more research should use an RCT design. On the other hand, since the evaluation design with experimental and control groups can contradict the basic premise of CBPR that emphasises the context and participation of the community, a lagged intervention used in Schulz et al. [42] may be considered to solve this issue.

As examined in our review, CBPR-based CVD management programmes showed positive effects on clinical, behavioural, cognitive, relational, and psychological aspects. In particular, Ralston et al. [30-32] showed that there is a longer effect in the experimental group than in the control group through repeated measurements after an intervention with an RCT design. However, while CPBR emphasises strengthening community capacity by allowing community residents to participate in decisions that affect their own health and exert influence on the quality of life of the entire community by establishing cooperative partnerships, securing sufficient resources, and promoting active participation and leadership of community members [57], none of the selected studies in our review measured the effects on community capacity. Specifically, measuring the effectiveness should not end with a one-time measurement immediately after the intervention; instead, it should evaluate the sustainability of an intervention and reflect the CBPR evaluation criteria suggested by Plumb et al. [58], including cooperation of community, group dynamics, and evaluation of community participation.

This scoping review for the key elements and effects of CBPR-based CVD management programmes over the last 10 years examined the recent trends in such programmes. Our results examined the sustainability and applicability of the CBPR-based CVD management programme by allowing community partnerships to understand the health needs of community residents, develop and implement programmes that are acceptable to community residents, and confirm for their effectiveness, instead of using the unilaterally provided standardised programmes.

This review has the following limitations: first, as our review included only studies published in English, most of the selected studies were conducted in North America, which did not reflect the cultural diversity of non-English speaking countries; and, second, with a focus on the development, implementation, and evaluation of CVD management programmes, problems such as obstacles and challenges arising in some processes of CBPR could not be emphasised.

\section{Conclusions}

We examined the key elements and effects of CBPR-based CVD management programmes using the scoping review method. Among the key elements of such programmes, there were many cases in which community organisations ( $\mathrm{CBO}$, FBOs) played the leading role in establishing partnerships and cases in which a decision-making committee was formed. Regarding the application of the CBPR principles, community partners rarely participated during the process of analysis and interpretation of research results and dissemination of research results to residents. Of the 17 studies in which the program effect were evaluated, all of the six RCT studies showed significantly positive effects in the experimental groups compared to the control groups.

It is necessary to develop strategies to improve the criteria based on which CBPR principles cannot be implemented during the development and application of a CBPR-based CVD management programme. In addition, more attempts to verify the effectiveness of high-quality research methodology should be made when evaluating the effectiveness of programmes.

\section{Declarations}

\section{Authors' contributions}

BP provided the main idea including research design of this study. BP and JY conceptualised the research questions, developed and agreed the search strategy, data extraction and synthesis, and conducted the search, screening, data extraction and analysis. All authors contributed to the manuscript, critically reviewed and approved the final manuscript.

\section{Funding}

This study was financially supported by National Research Foundation of Korea (No: 2020R1A2C1008591). The funding agency had no influence on the design, data collection, analysis, interpretation of data, or results of this study, nor on the writing of this manuscript.

\section{Availability of data and materials}

The datasets to be generated and analyzed in this study will not be publicly available and will be available by contacting the corresponding author on reasonable request. 


\section{Ethics approval and consent to participate}

Not applicable.

\section{Consent for publication}

Not applicable.

\section{Competing interests}

The authors declare no conflict of interests

\section{Authors details (optional)}

${ }^{1,2}$ Department of Nursing, Changwon National University, Gyeongnam, Republic of Korea.

\section{Abbreviations}

CVD: Cardiovascular disease

CBPR: Community-based participatory research

RCT: Randomised controlled trial

\section{References}

1. Go, A. S., Mozaffarian, D., Roger, V. L., Benjamin, E. J., Berry, J. D., ... Franco, S. Heart disease and stroke statistics-2014 update: a report from the American Heart Association. Circulation. 2014;129(3):e28.

2. Virani, S. S., Alonso, A., Benjamin, E. J., Bittencourt, M. S., Callaway, C. W., Carson, A. P., ... Djousse, L. Heart disease and stroke statistics-2020 update: a report from the American Heart Association. Circulation. 2020;E139-E596.

3. Stringhini S, Zaninotto P, Kumari M, Kivimäki M, Lassale C, Batty GD. Socio-economic trajectories and cardiovascular disease mortality in older people: the English Longitudinal Study of Ageing. Int J Epidemiol. 2018;47(1):36-46. doi:10.1093/ije/dyx106

4. Sung, J., Song, Y. M., \& Hong, K. P. Relationship between the shift of socioeconomic status and cardiovascular mortality. European journal of preventive cardiology. 2020;27(7):749-757.

5. Kim, Y. J., Lee, J. S., Park, J., Choi, D. S., Kim, D. M., Lee, K. H., ... Lee, J. Trends in socioeconomic inequalities in five major risk factors for cardiovascular disease in the Korean population: a cross-sectional study using data from the Korea National Health and Nutrition Examination Survey, 2001-2014. BMJ open. 2017;7(5):e014070.

6. Podolecka, E., Doryńska, A., Nadrowski, P., Skrzypek, M., Kwaśniewska, M., Drygas, W., ... Kozakiewicz, K. Socioeconomic status and cardiovascular risk SCORE. Kardiol Pol. 2018;76(3):560-565.

7. Healthy People 2020. Social Determinants of Health. Retrieved from https://www.healthypeople.gov/2020/topics-objectives/topic/social-determinants-ofhealth. 2017

8. Watson, K. B., Harris, C. D., Carlson, S. A., Dorn, J. M., \& Fulton, J. E. Disparities in adolescents' residence in neighborhoods supportive of physical activityUnited States, 2011-2012. Morbidity and Mortality Weekly Report. 2016;65(23):598-601.

9. McElfish, P. A., Ayers, B. L., Purvis, R. S., Long, C. R., Esquivel, M., \& Steelman, S. C. Best practices for community-engaged participatory research with Pacific Islander communities in the USA and USAPI: protocol for a scoping review. BMJ open. 2018;8(1).

10. Addison, C. C., Campbell Jenkins, B. W., Odom, D., Fortenberry, M., Wilson, G., Young, L., \& Antoine-LaVigne, D. Building collaborative health promotion partnerships: The Jackson heart study. International journal of environmental research and public health. 2016;13(1):25.

11. Williamson, G. R., Bellman, L., \& Webster, J. Action research in nursing and healthcare. Sage;2011.

12. Yoo, S. H. Using community-based participatory research (CBPR) for health promotion. Korean Journal of Health Education and Promotion. 2009;26(1):141-158.

13. Cook, W. K. Integrating research and action: a systematic review of community-based participatory research to address health disparities in environmental and occupational health in the USA. Journal of Epidemiology \& Community Health. 2008;62(8):668-676.

14. Marmot, M. Social determinants of health inequalities. The lancet. 2005;365(9464):1099-1104. 
15. Havranek, E. P., Mujahid, M. S., Barr, D. A., Blair, I. V., Cohen, M. S., Cruz-Flores,S., ... Yancy, C. W. Social determinants of risk and outcomes for cardiovascular disease:a scientific statement from the American Heart Association. Circulation. 2015;132(9):873-898.

16. Mohamed, E. A., Giama, N. H., Shaleh, H. M., Kerandi, L., Oseini, A. M., Ahmed Mohammed,H., ... Roberts, L. R. Knowledge, attitudes, and behaviors of viral hepatitis among recent African immigrants in the United States: a community based participatory research qualitative study. Frontiers in public health. 2020;8:25.

17. Manjunath, C., Ifelayo, O., Jones, C., Washington, M., Shanedling, S., Williams, J., \& Brewer, L. C. Addressing cardiovascular health disparities in minnesota: Establishment of a community steering committee by FAITH!(fostering african-american improvement in total health). International journal of environmental research and public health. 2019;16(21):4144.

18. Habila, M. A., Mantina, N., Kimaru, L. J., Musa, J., Ingram, M., \& Sagay, A. Community Engaged Approaches to Cervical Cancer Prevention and Control in Sub-Saharan Africa: A Scoping Review Protocol. 2020.

19. McElfish, P. A., Yeary, K., Sinclair, K. A., Steelman, S., Esquivel, M. K., Aitaoto,N., ... Ayers, B. L. Best Practices for Community-Engaged Research with Pacific Islander Communities in the US and USAPI: A Scoping Review. Journal of health care for the poor and underserved. 2019;30(4):1302.

20. Arksey, H., \& O'Malley, L. Scoping studies: towards a methodological framework. International journal of social research methodology. 2005;8(1):19-32.

21. Levac, D., Colquhoun, H., \& O'Brien, K. K. Scoping studies: advancing the methodology. Implementation science. 2010;5(1):1-9.

22. Tricco, A. C., Lillie, E., Zarin, W., O'Brien, K. K., Colquhoun, H., Levac, D., ...Straus, S. E. PRISMA extension for scoping reviews (PRISMA-ScR): checklist and explanation.Annals of internal medicine. 2018;169(7):467-473.

23. Balcazar, H., Rosenthal, L., De Heer, H., Aguirre, M., Flores, L., Vasquez, E., ...Schulz, L. Use of community-based participatory research to disseminate baseline results from a cardiovascular disease randomized community trial for Mexican Americans living in a US-Mexico border community. Education for health (Abingdon, England). 2009;22(3):279.

24. Balcázar, H. G., de Heer, H., Rosenthal, L., Duarte, M. O., Aguirre, M., Flores, L.,... Schulz, L. O. Peer reviewed: a Promotores de Salud intervention to reduce cardiovascular disease risk in a high-risk Hispanic border population, 2005-2008. Preventing chronic disease. 2010;7(2).

25. Balcázar, H., Wise, S., Rosenthal, E. L., Ochoa, C., Duarte-Gardea, M., Rodriguez,J., ... Hernandez, L. An ecological model using promotores de salud to prevent cardiovascular disease on the US-Mexico border: the HEART project. Preventing chronic disease. 2012;9.

26. Brewer, L. C., Balls-Berry, J. E., Dean, P., Lackore, K., Jenkins, S., \& Hayes, S. N. Fostering African-American improvement in total health (FAITH!): an application of the American Heart Association's Life's Simple $7^{\text {TM }}$ among Midwestern African-Americans. Journal of racial and ethnic health disparities. 2017;4(2):269-281

27. Brewer, L. C., Hayes, S. N., Caron, A. R., Derby, D. A., Breutzman, N. S., Wicks,A., ... Patten, C. A. Promoting cardiovascular health and wellness among African-Americans:Community participatory approach to design an innovative mobile-health intervention.PloS one. 2019;14(8):e0218724.

28. Brewer, L. C., Hayes, S. N., Jenkins, S. M., Lackore, K. A., Breitkopf, C. R., Cooper, L. A., \& Patten, C. A. Improving cardiovascular health among AfricanAmericans through mobile health: the faith! APP pilot study. Journal of General Internal Medicine. 2019;34(8):1376-1378.

29. Brewer, L. C., Hayes, S. N., Jenkins, S. M., Lackore, K. A., Breitkopf, C. R., Cooper, L. A., \& Patten, C. A. Improving cardiovascular health among AfricanAmericans through mobile health: the faith! APP pilot study. Journal of General Internal Medicine. 2019;34(8):1376-1378.

30. Ralston, P. A., Lemacks, J. L., Wickrama, K. K., Young-Clark, I., Coccia, C., Ilich,J. Z., ... O'Neal, C. W. Reducing cardiovascular disease risk in mid-life and older African Americans: A church-based longitudinal intervention project at baseline. Contemporary clinical trials. 2014;38(1):69-81.

31. Ralston, P. A., Young-Clark, I., \& Coccia, C. The development of health for hearts united: A longitudinal church-based intervention to reduce cardiovascular risk in mid-life and older African Americans. Ethnicity \& disease. 2017;27(1):21.

32. Ralston, P. A., Wickrama, K. K., Coccia, C. C., Lemacks, J. L., Young-Clark, I. M., \& Ilich, J. Z. Health for Hearts United Longitudinal Trial: Improving Dietary Behaviors in Older African Americans. American journal of preventive medicine. 2020;58(3):361-369.

33. Viswanathan, M., Ammerman, A., Eng, E., Garlehner, G., Lohr, K. N., Griffith, D.,... Whitener, L. Community-based participatory research: Assessing the evidence: Summary.AHRQ evidence report summaries. 2004.

34. Chen, P. G., Diaz, N., Lucas, G., \& Rosenthal, M. S. Dissemination of results in community-based participatory research. American journal of preventive medicine. 2010;39(4):372-378.

35. Bradley, H. A., \& Puoane, T. Prevention of hypertension and diabetes in an urban setting in South Africa: participatory action research with community health workers. 2007.

36. Delisle, A. T., Delisle, A. L., Chaney, B. H., Stopka, C. B., \& Northcutt, W. Methods for fostering a community academic partnership in a firefighter community. American journal of health behavior. 2013;37(6):721-733.

37. Lynch, E., Emery-Tiburcio, E., Dugan, S., White, F. S., Thomason, C., Jenkins, L.,... Ragland, A. Results of ALIVE: A faith-based pilot intervention to improve diet among African American church members. Progress in community health partnerships: research,education, and action. 2019;13(1):19-30.

38. Mudd-Martin, G., Martinez, M. C., Rayens, M. K., Gokun, Y., \& Meininger, J. C. Peer Reviewed: Sociocultural Tailoring of a Healthy Lifestyle Intervention to Reduce Cardiovascular Disease and Type 2 Diabetes Risk Among Latinos. Preventing chronic disease. 2013;10.

39. Kim, S., Koniak-Griffin, D., Flaskerud, J. H., \& Guarnero, P. A. The impact of lay health advisors on cardiovascular health promotion: using a communitybased participatory approach. Journal of Cardiovascular Nursing. 2004;19(3):192-199.

40. Rorie, J. A. L., Smith, A., Evans, T., Horsburgh, C. R., Brooks, D. R., Goodman, R.,... Geller, A. Using resident health advocates to improve public health screening and follow-up among public housing residents, Boston. 2010;2007-2008.

41. Pazoki, R., Nabipour, I., Seyednezami, N., \& Imami, S. R. Effects of a community-based healthy heart program on increasing healthy women's physical activity: a randomized controlled trial guided by Community-based Participatory Research (CBPR). BMC public health. 2007;7(1):1-8.

Page $16 / 18$ 
42. Schulz, A. J., Israel, B. A., Mentz, G. B., Bernal, C., Caver, D., DeMajo, R., ... Woods,S. Effectiveness of a walking group intervention to promote physical activity and cardiovascular health in predominantly non-Hispanic black and Hispanic urban neighborhoods:findings from the walk your heart to health intervention. Health Education \& Behavior.2015;42(3):380-392.

43. Zoellner, J., Connell, C., Madson, M. B., Thomson, J. L., Landry, A. S., Molaison,E. F., ... Yadrick, K. HUB city steps: a 6-month lifestyle intervention improves blood pressure among a primarily African-American community. Journal of the Academy of Nutrition and Dietetics. 2014;114(4):603-612.

44. Chimberengwa, P. T., \& Naidoo, M. description of community-based participatory research of hypertension awareness, prevention and treatment in a district of Matabeleland South Province, Zimbabwe. African journal of primary health care \& family medicine. 2019;11(1):1-9.

45. Skolarus, L. E., Cowdery, J., Dome, M., Bailey, S., Baek, J., Byrd, J. B., ... Brown,D. L. Reach out churches: A community-based participatory research pilot trial to assess the feasibility of a mobile health technology intervention to reduce blood pressure among African Americans. Health promotion practice. 2018;19(4):495-505.

46. Jayaprakash, M., Puri-Taneja, A., Kandula, N. R., Bharucha, H., Kumar, S., \& Dave, S. S. (2016). Qualitative process evaluation of a community-based culturally tailored lifestyle intervention for underserved South Asians. Health promotion practice. 2016;17(6):802-813.

47. Daudt, H. M., van Mossel, C., \& Scott, S. J. (2013). Enhancing the scoping study methodology: a large, inter-professional team's experience with Arksey and O'Malley's framework. BMC medical research methodology. 2013;13(1):1-9.

48. Seo, H. J., \& Kim, S. Y. What is scoping review?. Journal of Health Technology Assessment. 2018;6(1):16-21.

49. National Institutes of Health. Funding opportunity announcement: Community-Based Participatory Research at NIMH (R21). 2006. Retrieved from from: http://grants.nih.gov/grants/guide/pa-files/PAR-07-004.html

50. Minkler, M. Community-based research partnerships: challenges and opportunities. Journal of urban health. 2005;82(2):ii3-ii12.

51. Yoo, S. H. Community Participation for Health Promotion: Definitions and Applications. Korean Journal of Health Education and Promotion. 2012;29(4):57-66.

52. Ioannidis, J. P. Materializing research promises: opportunities, priorities and conflicts in translational medicine. Journal of Translational Medicine. 2004;2(1):1-6.

53. Prevention Research Center. Description of the logic model for the Prevention Research Centers (PRC) Program. 2008. Retrieved from Available from: https://www.cdc.gov/prc/about-prc-program/

54. Prevention Research Center. Policy statement for Core Research Projects. 2006. Retrieved from: https://www.cdc.gov/publichealthgateway/policy/cdcoffices-programs/

55. Public Health England. Community-Centred Public Health: Taking a Whole System Approach. 2020.

56. Bench, S., Day, T., \& Metcalfe, A. Randomised controlled trials: An introduction for nurse researchers. Nurse Researcher. 2013;20(5).

57. Giachello, A. L., Arrom, J. O., Davis, M., Sayad, J. V., Ramirez, D., Nandi, C., ...Coalition, C. S. D. C. A. Reducing diabetes health disparities through community-based participatory action research: the Chicago Southeast Diabetes Community Action Coalition.Public Health Reports. 2003;118(4): 309.

58. Plumb, M., Collins, N., Cordeiro, J. N., \& Kavanaugh-Lynch, M. Assessing process and outcomes: Evaluating community-based participatory research. Progress in Community Health Partnerships: Research, Education, and Action. 2008;2(2): 87-97.

59. Bess, K. D., Frerichs, L., Young, T., Corbie-Smith, G., Dave, G., Davis, K., ... Cene,C. W. Adaptation of an evidence-based cardiovascular health intervention for rural African Americans in the Southeast. Progress in community health partnerships: research,education, and action. 2019;13(4):385-396.

60. Pinsker, E. A., Enzler, A. W., Hoffman, M. C., Call, K. T., Amos, S., Babington-Johnson, A., \& Okuyemi, K. S. Peer Reviewed: A Community-Driven Implementation of the Body and Soul Program in Churches in the Twin Cities, Minnesota, 2011-2014. Preventing chronic disease; 2017:14.

61. Schroeder, K., Ratcliffe, S. J., Perez, A., Earley, D., Bowman, C., \& Lipman, T. H. Dance for health: an intergenerational program to increase access to physical activity. Journal of pediatric nursing. 2017;37:29-34.

62. Folta, S. C., Paul, L., Nelson, M. E., Strogatz, D., Graham, M., Eldridge, G. D.,... Seguin-Fowler, R. A. Changes in diet and physical activity resulting from the Strong Hearts, Healthy Communities randomized cardiovascular disease risk reduction multilevel intervention trial. International Journal of Behavioral Nutrition and Physical Activity.2019;16(1):1-12.

63. Perry, C. K., McCalmont, J. C., Ward, J. P., Menelas, H. D. K., Jackson, C., De Witz,J. R., ... Seguin, R. A. Mujeres Fuertes y Corazones Saludables: adaptation of the StrongWomen-healthy hearts program for rural Latinas using an intervention mapping approach. BMC public health. 2017;17(1):1-8.

\section{Figures}




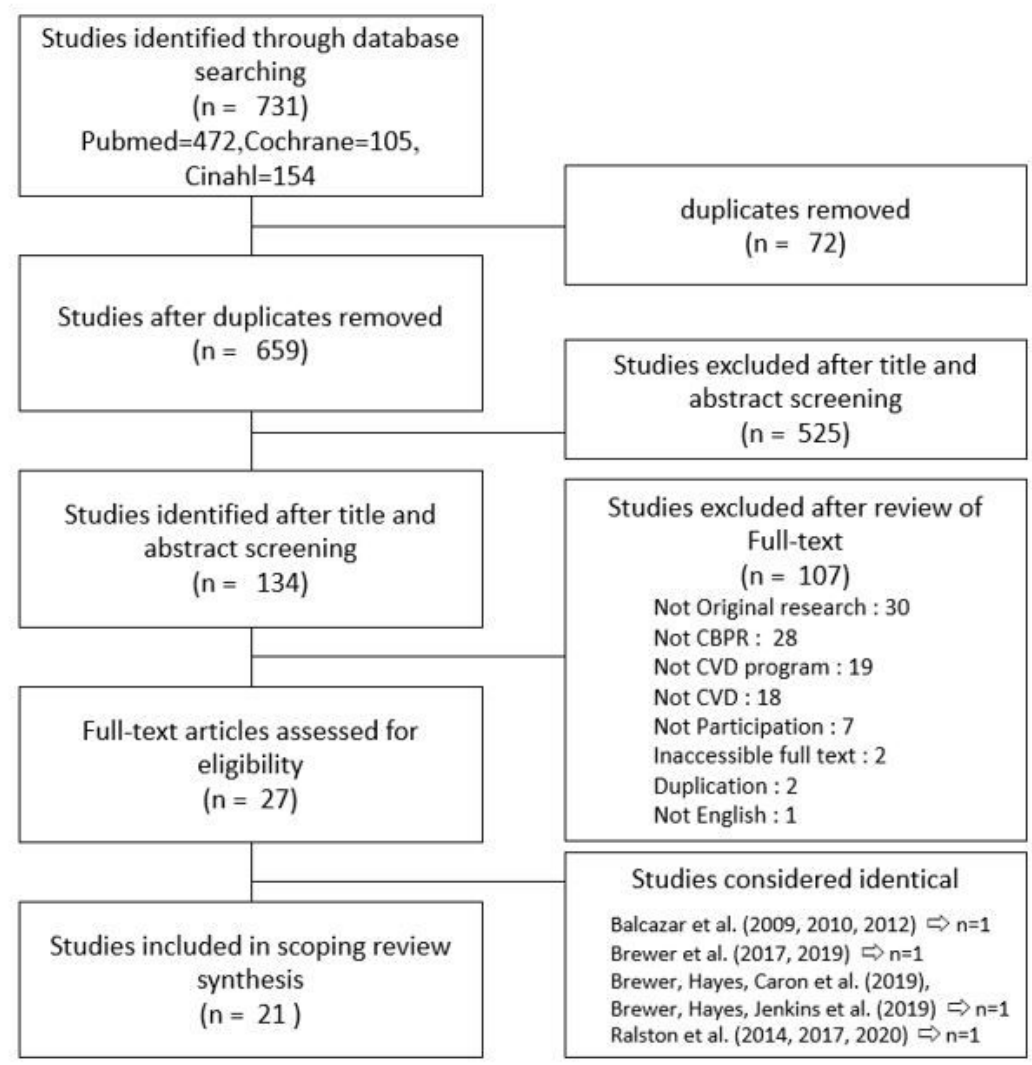

\section{Figure 1}

The sequential process of identifying the studies included in the analysis 